\title{
Frequency Driven Phasic Shifting and Elastic-Hysteretic Partitioning Properties of Fractional Mechanical System Representation Schemes
}

\author{
Jerzy T. Sawicki \\ Cleveland State University, j.sawicki@csuohio.edu \\ Joe Padovan \\ University of Akron
}

Follow this and additional works at: https://engagedscholarship.csuohio.edu/enme_facpub

Part of the Mechanical Engineering Commons

How does access to this work benefit you? Let us know!

\section{Publisher's Statement}

NOTICE: this is the author's version of a work that was accepted for publication in Journal of the Franklin Institute. Changes resulting from the publishing process, such as peer review, editing, corrections, structural formatting, and other quality control mechanisms may not be reflected in this document. Changes may have been made to this work since it was submitted for publication. A definitive version was subsequently published in Journal of the Franklin Institute, 336, 3, (04-01-1999); 10.1016/S0016-0032(98)00036-2

\section{Original Citation}

Sawicki, J.T. and Padovan, J. (1999) Frequency Driven Phasic Shifting and Elastic-Hysteretic Partitioning Properties of Fractional Mechanical System Representation Schemes. Journal of the Franklin Institute, 336(3), 423-433, doi: 10.1016/S0016-0032(98)00036-2.

This Article is brought to you for free and open access by the Mechanical Engineering Department at EngagedScholarship@CSU. It has been accepted for inclusion in Mechanical Engineering Faculty Publications by an authorized administrator of EngagedScholarship@CSU. For more information, please contact library.es@csuohio.edu. 


\title{
Frequency driven phasic shifting and elastic- hysteretic partitioning properties of fractional mechanical system representation schemes
}

\author{
Jerzy T. Sawickia,*, Joe Padovan ${ }^{\mathrm{b}}$ \\ a Department of Mechanical Engineering, Cleveland State University, Cleveland, $\mathrm{OH} 44115-2425$, USA \\ ${ }^{b}$ Department of Mechanical Engineering, The University of Akron, Akron, $\mathrm{OH} 44325-3903$, USA
}

\section{Introduction}

In recent years, increasingly exotic material combinations have been employed in structural applications to damp out vibrations and acoustical inputs. This includes a variety of metal-elastomer pairings in sandwiched and coating type configurations. The modeling of such hysteretic systems is often complicated by their frequency dependent damping characteristics. While such behavior can be simulated by

* Corresponding author. 
generalized Kelvin Voigt (KV) type representations [1,2], this generally yields very stiff models involving higher order derivatives [3]. Fractional formulations [3-8] can be used to bypass such difficulties. This follows from the fact that complete operator bases can be formed by irrational power sets bound by lower orders, i.e., $(0 ; 1),(0 ; 2), \ldots$ etc. $[3,6,7]$. While fractional operators $[3-13]$ yield improved model representations of system behavior, their earlier use was hampered by: (1) a lack of an understandable physical interpretation, and (2) somewhat complex computational properties.

Recently, improved computational schemes $[8,14]$ have been developed and benchmarked. Nonetheless, their further use is still hampered by a lack of physical insight. In the context of a lumped parameter system model, this paper investigates the manner in which fractional operators partition conservative and nonconservative behavior. Special emphasis is given to ascertain the manner in which the fractional order and frequency are involved in the partitioning process. In the sections which follow, detailed discussions are given on the governing equations, the asymptotic large time properties of fractional operators, the steady state solution, system energetics and importantly on the conservative-nonconservative energy splitting.

\section{Governing equations}

For mechanical systems with complex frequency dependent hysteresis, the governing equations of motion can be written in the form:

$$
\underline{\underline{M}} D_{2}(\underline{x})+\underline{F}(\underline{x}, \underline{Q})=\underline{G},
$$

where $\underline{M}, \underline{F}, \underline{G}, \underline{x} . \underline{Q}$ and $D_{2}(\cdot)$ respectively represent the mass matrix, restoring force vector, exciting force vector, displacement vector, the vector defining integro-differential operator powers, and the 2 nd time derivative. Assuming that $\underline{F}$ is a linear superposition of integro-differential operators, it follows that

$$
\underline{F}(\underline{x}, \underline{Q})=\sum_{r} \underline{\underline{K}}_{r} D_{Q(r)}(\underline{x}),
$$

where $\underline{\underline{K}}_{r}$ is the $r$ th material coefficient matrix, $Q(r)$ is the $r$ th element of $\underline{Q}$ and $D_{Q(r)}(\cdot)$ is the Louiville-Riemann operator, namely [15]

$$
D_{Q(r)}(\underline{x})=\frac{1}{\Gamma(-Q(r))} \int_{0}^{t} \frac{1}{(t-\tau)^{Q(r)+1}} \underline{x}(\tau) \mathrm{d} \tau .
$$

Under spectrally rich exciting forces, $\underline{G}$ can be written in the form

$$
\underline{G}(t)=\sum_{\ell} \mathrm{e}^{\mathrm{i} \omega_{\ell} t} \underline{G}_{\ell}
$$

where

$$
\underline{G}_{\ell}=\underline{\bar{G}}_{-\ell}
$$


such that (-) denotes complex conjugation. The components $\underline{G}_{\ell}$ and its pair $\underline{G}_{\ell}$ define both the amplitude and phasing of the input for each $\omega_{\ell}$ frequency.

Given that $Q(\ell)$ can range the real number axis, through Eq. (3), Eq. (2) can consist of fractional/integer integrals or derivatives. For the integer version, we obtain the classic Kelvin Voigt (KV) representation [1,2], i.e.,

$$
\underline{F}(\underline{x})={ }_{r} \underline{\underline{K}}_{r} D_{r}(\underline{x}) .
$$

\section{Asymptotic large time properties of fractional operators}

Given the use of Fourier decomposition (FFT) analysis, generally the steady state input to vibratory systems can be cast in terms of harmonic or damped harmonic functions involving phase shifts. This includes such functions as $\mathrm{e}^{\mathrm{j} t}, \mathrm{e}^{\alpha+\mathrm{j} t}$, $\cos (\omega t+\phi)$, and so on. As will be seen in this section, under such functions, the Louiville-Riemann operator $D_{Q(\ell)}(\cdot)$ can be replaced by a frequency and $Q$ weighed expression involving integer operators.

Based on the Louiville-Riemann definition, it can be shown that [15]

$$
D_{Q}\left(\mathrm{e}^{\mathrm{j} t}\right) \equiv(t) Q^{Q} \mathrm{e}^{\mathrm{j} t} \gamma^{*}(-Q, \mathrm{j} \omega t),
$$

where $\gamma^{*}(-Q, \mathrm{j} \omega t)$ defines the incomplete gamma function [16]. The incomplete gamma function has an asymptotic expansion which permits us to write [16]

$$
\frac{\gamma^{*}(-Q, \mathrm{j} \omega t)}{t^{Q}} \sim(\mathrm{j} \omega)^{Q}-\frac{\mathrm{e}^{\mathrm{j} t}}{\mathrm{j} \omega \Gamma(-Q)(t)^{Q+1}}\left[1-\frac{Q+1}{\mathrm{j} \omega t}+\mathrm{O}\left((\mathrm{j} \omega t)^{-2}\right)\right],
$$

where $\Gamma(-Q)$ is the gamma function [16]. Based on Eqs. (7) and (8) it follows that asymptotically (i.e., $t \rightarrow \infty$ )

$$
D_{Q}\left(\mathrm{e}^{\mathrm{j} t}\right) \sim(\mathrm{j} \omega)^{Q} \mathrm{e}^{\mathrm{j} t}-\frac{1}{\mathrm{j} \omega \Gamma(-Q)(t)^{Q+1}}\left[1-\frac{Q+1}{\mathrm{j} \omega t}+\mathrm{O}\left((\mathrm{j} \omega t)^{-2}\right)\right] .
$$

Similar expressions can be derived for $\cos (\omega t)$ and $\sin (\omega t)$.

In terms of Eq. (9), for $t \gg 0$, the following asymptotic expression can be obtained, i.e.,

$$
\begin{aligned}
& D_{Q}\left(\mathrm{e}^{\mathrm{j} t}\right) \sim(\omega)^{Q} \mathrm{e}^{\mathrm{j}(t+(\pi / 2) Q)}+\mathrm{O}\left(\frac{1}{t^{1+Q}}\right), \\
& D_{Q}(\cos (\omega t)) \sim(\omega)^{Q} \cos \left(\omega t+\frac{\pi}{2} Q\right)+\mathrm{O}\left(\frac{1}{t^{1+Q}}\right), \\
& D_{Q}(\sin (\omega t)) \sim(\omega)^{Q} \sin \left(\omega t+\frac{\pi}{2} Q\right)+\mathrm{O}\left(\frac{1}{t^{2+Q}}\right) .
\end{aligned}
$$


Based on the form of the double angle identities, Eqs. (10-12) can be rewritten in the form:

$$
\begin{aligned}
D_{Q}\left(\mathrm{e}^{\mathrm{j} t} ; \cos (\omega t) ; \sin (\omega t)\right)= & (\omega)^{Q}\left\{\cos \left(\frac{-}{2} Q\right)\left[\mathrm{e}^{\mathrm{j} t} ; \cos (\omega t) ; \sin (\omega t)\right]\right. \\
& \left.+\sin \left(\frac{-}{2} Q\right)\left[\mathrm{je}^{\mathrm{j} t} ;-\sin (\omega t) ; \cos (\omega t)\right]\right\} .
\end{aligned}
$$

Since $\mathrm{e}^{\mathrm{j} t}, \cos (\omega t)$, and $\sin (\omega t)$ form complete bases, any harmonic exciting force can be written in the form

$$
\begin{aligned}
& H\left(\underline{\omega}^{\prime} t\right)={ }_{i} h_{i}\left(\mathrm{e}^{\mathrm{j}}{ }^{i} t ; \cos \left(\omega_{i} t+\phi_{i}\right) ; \sin \left(\omega_{i} t+\gamma_{i}\right)\right), \\
& \underline{\omega}^{\prime} t \equiv\left(\omega_{1} t, \omega_{2} t, \ldots, \omega_{i} t \ldots\right) .
\end{aligned}
$$

Given that each member of Eq. (14) satisfies Eq. (13), it follows that for $t \gg 0$,

$$
\lim _{t \gg 0} D_{Q}\left\{H\left(\underline{\omega}^{\prime} t\right)\right\} \equiv\left\{\left(\omega_{i}\right)^{Q} \cos (\overline{2} Q) h_{i}\left(\omega_{i} t\right)+\left(\omega_{i}\right)^{Q-1} \sin \left(\frac{-}{2} Q\right) D_{1}\left(h_{i}\left(\omega_{i} t\right)\right)\right\}
$$

wherein $h_{i}\left(\omega_{i} t\right)$ is any harmonic function. Equation (16) applies for essentially all $Q$. When $Q$ is an integer, then the classical results are retrieved, namely

$$
\begin{aligned}
& \lim _{Q \rightarrow 0} D_{Q}\left\{H\left(\underline{\omega}^{\prime} t\right)\right\} \rightarrow H\left(\underline{\omega}^{\prime} t\right), \\
& \lim _{Q=1} D_{Q}\left\{H\left(\underline{\omega}^{\prime} t\right)\right\} \rightarrow D_{1}\left(H\left(\underline{\omega}^{\prime} t\right)\right) .
\end{aligned}
$$

For non-integer $Q$, as noted earlier, $D_{Q}(H)$ is a frequency and $Q$ weighted superposition of $H$ and $D_{1}(H)$.

The phasic behaviour of $D_{Q}$ follows directly from Eqs. (10)-(12) and Eq. (14), that is

$$
\lim _{t \gg 0} D_{Q}\left\{H\left(\underline{\omega}^{\prime} t\right)\right\} \rightarrow{ }_{i}\left(\omega_{i}\right)^{Q} h_{i}\left(\omega_{i} t+\frac{-}{2} Q\right) .
$$

Since $Q$ may be an integer, rational or irrational number, the most direct interpretation of $h_{i}\left(\omega_{i} t+(/ 2) Q\right)$ follows from Eq. (16), namely that

$$
h_{i}\left(\omega_{i} t+\frac{-}{2} Q\right)=\cos \left(\frac{-}{2} Q\right) h_{i}\left(\omega_{i} t\right)+\frac{1}{\omega_{i}} \sin \left(\frac{-}{2} Q\right) D_{1}\left(h_{i}\left(\omega_{i} t\right)\right) .
$$

\section{Steady state solutions}

For $t \gg 0$, general system hysteresis induces the so-called steady state response which involves a harmonic type behavior. In this context, under the spectrally rich system exciting field defined by Eq. (4), the particular-steady state-solution to Eq. (1) can be written in the form

$$
\underline{x}=\mathrm{e}^{\mathrm{j}}{ }^{i t} \underline{x}_{i}
$$


Based on the asymptotic differentiability properties defined by Eqs. (10) and (19), the coefficients of Eq. (21) take the form

$$
\underline{x}_{i}=\underline{\underline{\Psi}}_{i}{ }^{1} \underline{G}_{i},
$$

where

$$
\underline{\underline{\Psi}}_{i}=-\left(\omega_{i}\right)^{2} \underline{\underline{M}}+{ }_{i}\left(\omega_{i}\right)^{Q(r)} \mathrm{e}^{\mathrm{j}(/ 2) Q(r)} \underline{\underline{K}}_{r} .
$$

For the 1-D case, Eqs. (22) and (23) reduce to the simplified expression

$$
x_{i}=\frac{G_{i}}{-\left(\omega_{i}\right)^{2} M+{ }_{r}\left(\omega_{i}\right)^{Q(r)} \mathrm{e}^{\mathrm{j}(/ 2) Q(r)} K_{r}} .
$$

Note $\left(\underline{x}_{i} ; x_{i}\right)$ are complex numbers which form conjugate pairs with $\left(\underline{x}_{i} ; x_{i}\right)$. Because of this, the $\pm(i)$ th mode takes the form

$$
x=2\left\{\operatorname{Re}\left(x_{i}\right) \cos \left(\omega_{i} t\right)-\operatorname{Im}\left(x_{i}\right) \sin \left(\omega_{i} t\right)\right\}
$$

which casted in a phasic manner yields the expression

$$
x={ }_{i} A_{i} \cos \left(\omega_{i} t+\Xi_{i}\right)
$$

where

$$
\begin{aligned}
& A_{i}=2 \sqrt{\left(\operatorname{Re}\left(x_{i}\right)\right)^{2}+\left(\operatorname{Im}\left(x_{i}\right)\right)^{2}}, \\
& \Xi_{i}=\tan ^{1}\left(\frac{-\operatorname{Im}\left(x_{i}\right)}{\operatorname{Re}\left(x_{i}\right)}\right) .
\end{aligned}
$$

Considering the one mode KV model, Figure 1 illustrates the amplitude peak dependency on fractional order $Q$ over a range of frequencies. Figure 2 illustrates the amplitude magnitude behavior in the fractional order and frequency domain. As can be seen, for $Q<1$ the peak amplitude shifts to the right of the undamped reasonance while for $Q>1$, the peak shifts to the left. On the other hand, the strong influence of frequency on phasic behavior is shown in Fig. 3. Note the fact that for an integer derivatives, i.e., $Q=1$, the phase at resonance is fixed. In contrast, for the fractional form it shows the linear dependence on fractional order, i.e., $\Xi=\frac{1}{2} q$.

\section{Energetics}

A better appreciation of the mechanisms leading to the $Q$-dependent amplitude and phasic shifting can be ascertained through an understanding of the conservativenonconservative energy partitioning induced by the fractional operator representation. In particular, the rate of work done during system excursions can be estimated by the expression:

$$
D_{1}(W)=D_{1}\left(\underline{x}^{\prime}\right) \underline{F} .
$$




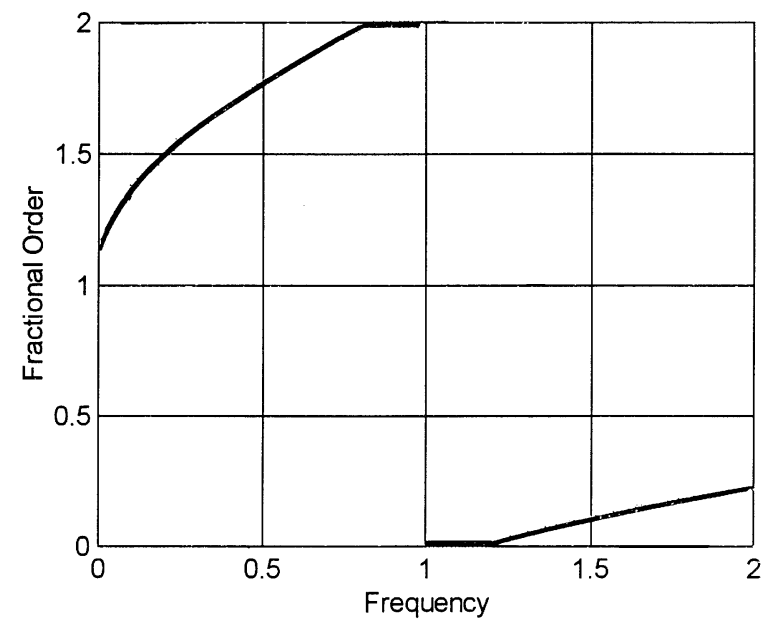

Fig. 1. Fractional order frequency dependency of peak amplitude: single mode KV model: $Q \in[0,2]$.

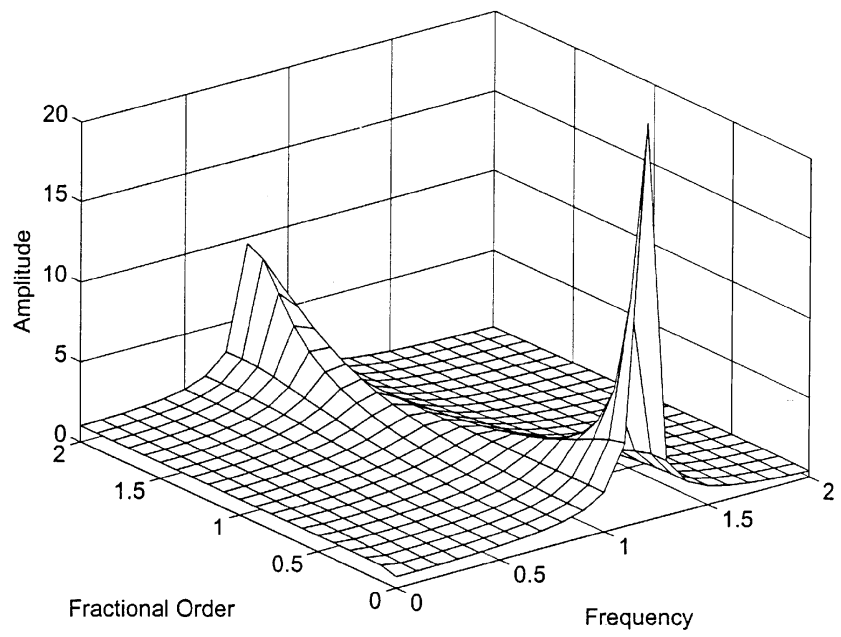

Fig. 2. Fractional order frequency dependency of amplitude magnitude: single mode KV model: $Q \in[0,2]$.

In terms of Eqs. (2) and (21), Eq. (29) yields the relation

$$
D_{1}(W)=\underset{r \quad \alpha \quad \beta}{ } \mathrm{j} \omega_{\alpha}\left(\omega_{\beta}\right)^{Q(r)} \mathrm{e}^{\mathrm{j}(/ 2) Q(r)} \Phi_{\alpha \beta r} \mathrm{e}^{\mathrm{j}\left(\alpha_{\alpha}^{+}{ }_{\beta}\right) t},
$$

where

$$
\Phi_{\alpha \beta r}=\underline{x}_{\alpha}^{\prime} \underline{\underline{K}}_{r} \underline{x}_{\beta} .
$$




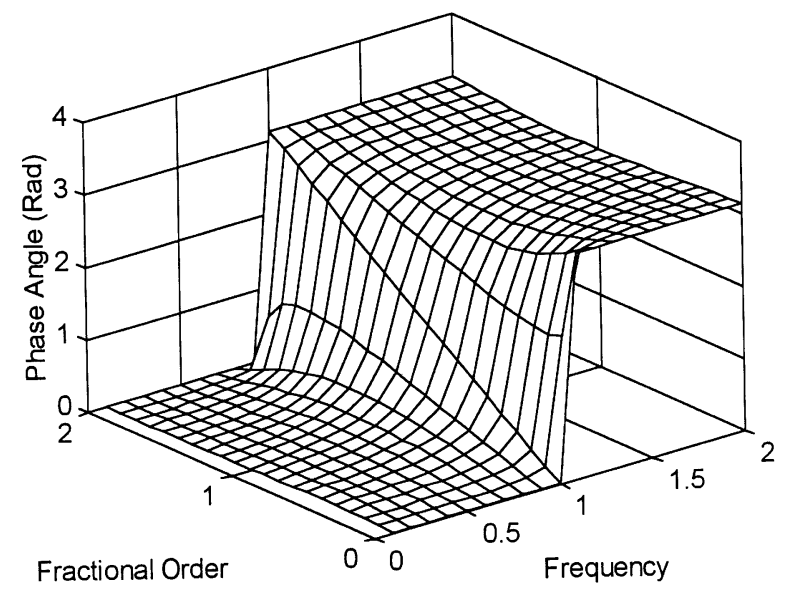

Fig. 3. Phasic behavior as a function of fractional order $Q \in[0,2]$ and frequency $\omega \in[0,2]$ : single mode KV model.

To determine the conservative-nonconservative partitioning, we introduce expressions for the rates of elastic energy storage and the traditional viscous energy dissipation. These are defined by

$$
\begin{aligned}
& D_{1}(E E S)=D_{1}\left(\underline{x}^{\prime}\right) \underline{K}_{r} \underline{x}, \\
& D_{1}(V E D)=D_{1}\left(\underline{x}^{\prime}\right) \underline{\underline{K}}_{r} D_{1}(\underline{x}) .
\end{aligned}
$$

Again based on Eq. (21), Eqs. (32) and (33) yield

$$
\begin{aligned}
& D_{1}(E E S)={ }_{\alpha \beta} D_{1}\left(E E S_{\alpha \beta}\right), \\
& D_{1}(V E D)={ }_{\alpha \quad \beta} D_{1}\left(V E D_{\alpha \beta}\right),
\end{aligned}
$$

where

$$
\begin{aligned}
& D_{1}\left(E E S_{\alpha \beta}\right)=j \omega_{\alpha} \underline{x}_{\alpha}^{\prime} \underline{\underline{K}}_{r} \underline{x}_{\beta} \mathrm{e}^{\mathrm{j}\left({ }_{\alpha}+{ }_{\beta}\right) t}, \\
& D_{1}\left(V E D_{\alpha \beta}\right)=-\omega_{\alpha} \omega_{\beta} \underline{x}_{\alpha}^{\prime} \underline{\underline{K}}_{r} \underline{\underline{x}}_{\beta} \mathrm{e}^{\mathrm{j}\left({ }_{\alpha}+{ }_{\beta}\right) t} .
\end{aligned}
$$

Now, employing Euler's identity, Eq. (30) can be recast as follows

$$
\begin{aligned}
D_{1}(W)= & \underset{r \alpha_{\beta}}{ }\left\{\mathrm{j} \omega_{\alpha}\left(\omega_{\beta}\right)^{Q(r)} \cos \left(\frac{-}{2} Q(r)\right)-\omega_{\alpha}\left(\omega_{\beta}\right)^{Q(r)} \sin \left({ }_{2} Q(r)\right)\right\} \\
& \times \Phi_{\alpha \beta r} \mathrm{e}^{\mathrm{j}\left({ }_{\alpha}+{ }_{\beta}\right) t} .
\end{aligned}
$$


By drawing an analogy between Eqs. (36) and (37) with the first and second partitions of Eq. (38) we can recast $D_{1}(W)$ in the form

$$
\begin{gathered}
D_{1}(W)=r_{r \alpha \beta}\left\{\left(\omega_{\beta}\right)^{Q(r)} \cos \left(\frac{-}{2} Q(r)\right) D_{1}\left(E E S_{\alpha \beta}^{(r)}\right)+\left(\omega_{\beta}\right)^{Q(r)-1}\right. \\
\left.\quad \times \sin (\overline{2} Q(r)) D_{1}\left(V E D_{\alpha \beta}^{(r)}\right)\right\}
\end{gathered}
$$

where

$$
\begin{aligned}
& D_{1}\left(E E S_{\alpha \beta}^{(r)}\right)=\mathrm{j} \omega_{\alpha} \Phi_{\alpha \beta r} \mathrm{e}^{\mathrm{j}\left(\alpha_{\alpha}+{ }_{\beta}\right) t}, \\
& D_{1}\left(V E D_{\alpha \beta}^{(r)}\right)=-\omega_{\alpha} \omega_{\beta} \Phi_{\alpha \beta r} \mathrm{e}^{\mathrm{j}\left({ }_{\alpha}+{ }_{\beta}\right) t} .
\end{aligned}
$$

Here $D_{1}\left(E E S_{\alpha \beta}^{(r)}\right)$ and $D_{1}\left(V E D_{\alpha \beta}^{(r)}\right)$, respectively provide time scaling for the $(r)$ th mode rates of elastic energy storage and dissipation. Noting Eq. (38), the $Q(r)$ functional embedding sets up partitioning between the said conservative and nonconservative features of the energetics.

For a single harmonic input, Eqs. (39)-(41) reduce to the following more tractable form, i.e.,

$$
D_{1}(W)={ }_{r}\left\{2(\omega)^{Q(r)} \cos \left({ }_{2} Q(r)\right) D_{1}\left(E E S^{(r)}\right)+2(\omega)^{Q(r)-1} \sin \left({ }_{2} Q(r)\right) D_{1}\left(V E D^{(r)}\right)\right\},
$$

where

$$
\begin{aligned}
& D_{1}\left(E E S^{(r)}\right)=\omega\left[-\operatorname{Im}\left(\Phi_{r}^{*}\right)-\operatorname{Im}\left(\Phi_{r}\right) \cos (2 \omega t)-\operatorname{Re}\left(\Phi_{r}\right) \sin (2 \omega t)\right], \\
& D_{1}\left(V E D^{(r)}\right)=(\omega)^{2}\left[\operatorname{Re}\left(\Phi_{r}^{*}\right)-\operatorname{Re}\left(\Phi_{r}\right) \cos (2 \omega t)+\operatorname{Im}\left(\Phi_{r}\right) \sin (2 \omega t)\right]
\end{aligned}
$$

with

$$
\Phi_{r}^{*(r)}=\underline{x}^{\prime} \underline{K}_{r} \underline{\bar{x}} .
$$

Given that all $\left[K^{(r)}\right] ; r \in[0,1, \ldots)$ are symmetrical, then

$$
\operatorname{Im}\left(\Phi_{r}^{*}\right) \equiv 0 \text { for all } r .
$$

Hence Eq. (43) can be recast as

$$
D_{1}\left(E E S^{(r)}\right)=-\omega\left[\operatorname{Im}\left(\Phi_{r}\right) \cos (2 \omega t)+\operatorname{Re}\left(\Phi_{r}\right) \sin (2 \omega t)\right] .
$$

In a phasic format, Eqs. (43) and (44) yield the expressions

$$
\begin{aligned}
& D_{1}\left(E E S^{(r)}\right)=-(\omega)\left[\operatorname{Im}\left(\Phi_{r}^{*}\right)+\Gamma_{r} \cos \left(2 \omega t+\theta_{1}\right)\right], \\
& D_{1}\left(V E D^{(r)}\right)=\left(\omega^{2}\right)\left[\operatorname{Re}\left(\Phi_{r}^{*}\right)+\Gamma_{r} \cos \left(2 \omega t+\theta_{2}\right)\right]
\end{aligned}
$$


with

$$
\begin{aligned}
& \theta_{1}=\tan ^{-1}\left(\operatorname{Im}\left(\Phi_{r}\right) / \operatorname{Re}\left(\Phi_{r}\right)\right), \\
& \theta_{2}=\tan ^{-1}\left(-\operatorname{Re}\left(\Phi_{r}\right) / \operatorname{Im}\left(\Phi_{r}\right)\right), \\
& \Gamma_{r}=\overline{\left(\operatorname{Re}\left(\Phi_{r}\right)\right)^{2}+\left(\operatorname{Im}\left(\Phi_{r}\right)\right)^{2} .}
\end{aligned}
$$

Alternatively, if we seek the net phasic behavior, we obtain the expression

$$
\begin{gathered}
D_{1}(W)={ }_{r} 2\left\{-(\omega)^{Q(r)+1} \operatorname{Im}\left(\phi_{r}^{*}\right) \cos \left(\frac{-}{2} Q(r)\right)(\omega)^{Q(r)+1} \operatorname{Re}\left(\phi_{r}^{*}\right) \sin \left(\frac{-}{2} Q(r)\right) \gamma_{r}\right. \\
\left.\cos \left(2 \omega t+\Phi_{N r}\right)\right\},
\end{gathered}
$$

where here

$$
\begin{aligned}
\gamma_{r}= & {\left[\left(\operatorname{Re}\left(\phi_{r}\right) \sin \left(\frac{}{2} Q(r)\right)+\operatorname{Im}\left(\phi_{r}\right) \cos \left(\frac{-}{2} Q(r)\right)\right)^{2}\right.} \\
& \left.+\left(-\operatorname{Re}\left(\phi_{r}\right) \cos \left(\frac{-}{2} Q(r)\right)+\operatorname{Im}\left(\phi_{r}\right) \sin \left(\frac{-}{2} Q(r)\right)\right)^{2}\right]^{1 / 2}, \\
\Phi_{N r}= & \tan ^{-1}\left\{\frac{-\operatorname{Im}\left(\phi_{r}\right) \cos ((/ 2) Q(r))-\operatorname{Re}\left(\phi_{r}\right) \sin ((/ 2) Q(r))}{\operatorname{Im}\left(\phi_{r}\right) \sin ((/ 2) Q(r))-\operatorname{Re}\left(\phi_{r}\right) \cos ((/ 2) Q(r))}\right\} .
\end{aligned}
$$

As $Q \equiv 0$ or 1

$$
\begin{aligned}
D_{1}(W(Q \equiv 0))= & \underset{r}{ }(\omega)^{Q(r)+1}\left\{-\operatorname{Im}\left(\phi_{r}^{*}\right)\right. \\
& \left.-\left[\left(\operatorname{Im}\left(\phi_{r}\right)\right)^{2}+\left(\operatorname{Re}\left(\phi_{r}\right)\right)^{2}\right]^{1 / 2} \cos \left(2 \omega t+\tan ^{1}\left(\frac{\operatorname{Im}\left(\phi_{r}\right)}{\operatorname{Re}\left(\phi_{r}\right)}\right)\right)\right\} \\
D_{1}(W(Q \equiv 1))= & 2_{r}(\omega)^{Q(r)+1}\left\{\operatorname{Re}\left(\phi_{r}^{*}\right)\right. \\
& \left.+\left[\left(\operatorname{Im}\left(\phi_{r}\right)\right)^{2}+\left(\operatorname{Re}\left(\phi_{r}\right)\right)^{2}\right]^{1 / 2} \cos \left(2 \omega t+\tan ^{1}\left(\frac{-\operatorname{Re}\left(\phi_{r}\right)}{\operatorname{Im}\left(\phi_{r}\right)}\right)\right)\right\} .
\end{aligned}
$$

Equations (39) and (42) define the level of partitioning of conservative and nonconservative effects over a range of $Q$ th ordered fractional operators. Considering the case of the single spectral input to a single fractional KV model, we obtain that

$$
\begin{aligned}
D_{1}(W)=\left(\chi^{2}\right) K_{0}\{ & -\frac{1}{2} \sin (2 \omega t+\phi)\left[\omega+\cos \left(\frac{-}{2} Q\right)(\omega)^{Q+1} \frac{K_{1}}{K_{0}}\right] \\
& \left.+\sin ^{2}(\omega t+\phi)(\omega)^{Q+1} \sin \left(\frac{-}{2} Q\right) \frac{K_{1}}{K_{0}}\right\},
\end{aligned}
$$


where

$$
\begin{aligned}
& \chi=G\left[\frac{1}{\left(K_{0}+(\omega)^{Q} K_{1} \cos \left(\frac{-}{2} Q\right)-\omega^{2} M\right)^{2}+\omega^{2 Q}\left(K_{1}\right)^{2} \sin ^{2}\left(\frac{-}{2} Q\right)}\right]^{1 / 2}, \\
& \phi=\tan ^{-1}\left[\frac{-(\omega)^{Q} K_{1} \sin ((/ 2) Q)}{K_{0}+(\omega)^{Q} K_{1} \cos ((/ 2) Q)-(\omega)^{2} M}\right] .
\end{aligned}
$$

Since

$$
D_{1}(W)=D_{1}(E E S)+D_{1}(V E D),
$$

then

$$
\begin{aligned}
& D_{1}(E E S)=-\frac{(\chi)^{2} K_{0}}{2}\left[\omega+\cos \left(\frac{-}{2} Q\right) \omega^{Q+1}\left(\frac{K_{1}}{K_{0}}\right)\right] \sin (2 \omega t+\phi), \\
& D_{1}(V E D)=\frac{(\chi)^{2} K_{0}}{2} \sin \left(-\frac{{ }^{2}}{2}\right) \omega^{Q+1}\left(\frac{K_{1}}{K_{0}}\right) \sin ^{2}(2 \omega t+\phi) .
\end{aligned}
$$

As can be seen from Eq. (63), $D_{1}(V E D)$ has a mixed definiteness contingent on the tuning of $Q$. For instance, for $0<Q<1$, it is positive definite for all $t$. Depending on the relative size of $K_{1}$ and $K_{0}, D_{1}(E E S)$ may also have a mixed definiteness for variations in $Q$. Regardless, as $t$ is varied in any given period, there is a storage mode wherein $D_{1}(E E S)>0$ and a relaxation mode such that $D_{1}(E E S)<0$.

Noting Eqs. (61)-(63), the conservative and nonconservative partitions are formed by a $Q$ weighted frequency dependent splitting. Recalling Section 4 , for $Q<1$, the peak amplitudes occurred to the left of resonance while for $Q>1$, the shift was to the right. Based on Eqs. (62) and (63), as $Q$ is varied from $1 \rightarrow 0$, the elastic storage process grows in intensity. In contrast, as $Q$ is varied from $0 \rightarrow 1$, the nonconservative, i.e., rate of dissipation becomes more intense. Varying $Q$ in the interval $(1,2)$ the process is reversed. This behavior is repeated every interval of 2 in $Q$ space. In the negative range, $Q<0$, the conservative/nonconservative dominance tends act analogously in the negative axis direction.

\section{Summary}

It follows from the preceding sections, that the fractional operator is actually a viscoelastic element which partitions the system energy into conservative and nonconservative components. The order of the operator, $Q$, serves to weight the splitting in favor of the nearest integer operator. Such properties enable the tuning of both amplitude and phasing. In this way the proper hysteresis, amplitude and phase-frequency behavior can be simulated by such models. These features give fractional integro-differential operators a decided advantage over their integer subset. 


\section{References}

[1] W. Flügge, Viscoelasticity, Blaisdell, New York, 1967.

[2] M. Simhambhatla, A.I. Leonov, The extended Pade-Laplace method for efficient discretization of linear viscoelastic spectra, Rheol. Acta 32 (1993) 584-600.

[3] J. Padovan, J.T. Sawicki, Diophantine type fractional derivative representation of structural hysteresis, Part I: Formulation, Comput. Mech. 19 (1997) 335-341.

[4] R.L. Bagley, R.A. Calico, Fractional order state equations for the control of viscoelastically damped structures, J. Guidance Control Dynamics 14 (1991) 304-311.

[5] R.L. Bagley, P.J. Torvik, Fractional calculus - A different approach to the analysis of viscoelastically damped structures, AIAA J. 21 (5) (1983) 741-748.

[6] J. Padovan, Y.H. Guo, General response of viscoelastic systems modeled by fractional operators, J. Franklin Inst. 325 (1988) 247-275.

[7] P.J. Torvik, R.L. Bagley, On the appearance of the fractional derivative in the behavior of real materials, ASME J. Appl. Mech. 51 (1984) 294-298.

[8] J. Padovan, Numerical analysis of discrete fractional integrodifferential structural dampers, J. Finite Elements Anal. Des. 3 (1987) 297-314.

[9] W. Smit, H. deVries, Rheological models containing fractional derivatives, Rheol. Acta 6 (1970) $525-534$.

[10] M. Caputo, F. Mairardi, A new dissipation model based on memory mechanism, Pure Appl. Geophys. 91 (1971) 134-147.

[11] M. Stiassnie, On the application of fractional calculus for the formulation of viscoelastic models, Appl. Math. Modelling 3 (1979) 300-302.

[12] L.C. Rogers, Operators and fractional derivatives for viscoelastic constitutive equations, J. Rheol. 27 (1983) 351-372.

[13] R.C. Koeller, Applications of fractional calculus to the theory of viscoelasticity, ASME J. Appl. Mech. 51 (1984) 299-307.

[14] M. Enelund, B.L. Josefson, Time-domain FE-analysis of viscoelastic structures having constitutive relations involving fractional derivatives, AIAA-96-1394-CP, 1996.

[15] K.S. Miller, B. Ross, An Introduction to the Fractional Calculus and Differential Equations, Wiley, New York, 1993.

[16] I.A. Stegun, M. Abramowitz, Handbook of Mathematical Functions: with Formulas, Graphs, and Mathematical Tables, Dover, New York, 1964.

Post-print standardized by MSL Academic Endeavors, the imprint of the Michael Schwartz Library at Cleveland State University, 2014 